\title{
Compliant MEMS Motion Characterization by Nanoindentation
}

\author{
Joseph Goerges Choueifati ${ }^{1}$, Craig Lusk ${ }^{1}$, Xialou Pang ${ }^{1,2}$, and Alex A. Volinsky ${ }^{1}$ \\ ${ }^{1}$ Mechanical Engineering, University of South Florida, Tampa, FL, 33620 \\ ${ }^{2}$ Department of Materials Physics and Chemistry, University of Science and Technology \\ Beijing, Beijing, 100083, China, People's Republic of
}

\begin{abstract}
Large out-of-plane displacements can be achieved when compliant mechanisms are utilized in MEMS. While mathematical and macroscopic modeling is helpful in building original designs, the actual MEMS device motion needs to be characterized in terms of the forces and displacements. A nanoindentation apparatus equipped with Berkovich diamond tip was used in an attempt to actuate and characterize the motion of the Bistable Spherical Compliant Micromechanism with a nonlinear (approximately cubic) mechanical response. Based on the obtained lateral force-displacement data it was concluded that the Berkovich diamond tip was too sharp, thus cutting through the polysilicon material of the MEMS device.
\end{abstract}

\section{INTRODUCTION}

The most common technique used in building MEMS is surface micromachining [1, 2] due its simplicity and low cost. A challenge in using surface micromachining is that it produces essentially two-dimensional products. The ratio of the length and width with respect to the thickness of the elements created is high, thus most MEMS have a planar working space, where the motion of their links traces a single plane [3]. In some applications such as active Braille [4] and micro-optical systems [5], it may be useful for MEMS to achieve accurate three-dimensional motion. This paper provides the results obtained from testing a bistable compliant MEMS device with out-of-plane motion using a nanoindenter. A description of the bistable spherical micromechanism is also presented.

Mechanisms that rely on elastic deformation of their flexural members to carry out mechanical tasks of transforming and transferring energy, force and motion are called compliant mechanisms [6]. Furthermore, compliant mechanisms combine energy storage and motion, thus eliminating the need for separate components of joints and springs [6]. Many products currently on the market such as nail clippers, shampoo cap hinges and mechanical pens utilize compliant segments in their designs. In addition, studies have shown that one of the main reasons behind MEMS failure is joints wear [7]; thus replacing rigid multi-pieces joints with compliant single member joints will likely increase the device's lifespan [8-10].

A bistable mechanism is a mechanism that has two stable equilibrium points within its range of motion. A mechanism is considered to be in stable equilibrium if it returns to its equilibrium position after being subjected to small forces or disturbances. A mechanism is in an unstable equilibrium when a small force causes the mechanism to change positions, usually to a position of stable equilibrium. According to Lagrange-Dirichlet theorem, an object is in a stable equilibrium when its potential energy is at its local minimum. We have designed a bistable device using compliant mechanism theory that has its first stable position in the plane of fabrication and its second stable position out of the fabrication plane. The first stable position of the mechanism is shown in Figure 1a and the second stable position of the mechanism is shown in Figure 1b. 


\section{EXPERIMENT}

The design of the MEMS device followed the rules set by the Multi-User MEMS Processes (MUMPs) chosen for fabrication. The MUMPs is a three-layer polysilicon surface micromachining process, which has the following features:

1) polysilicon is used as the structural material;

2) deposited oxide (PSG) is used as the sacrificial layer and silicon nitride is used as electrical isolation between the polysilicon and the substrate [11].
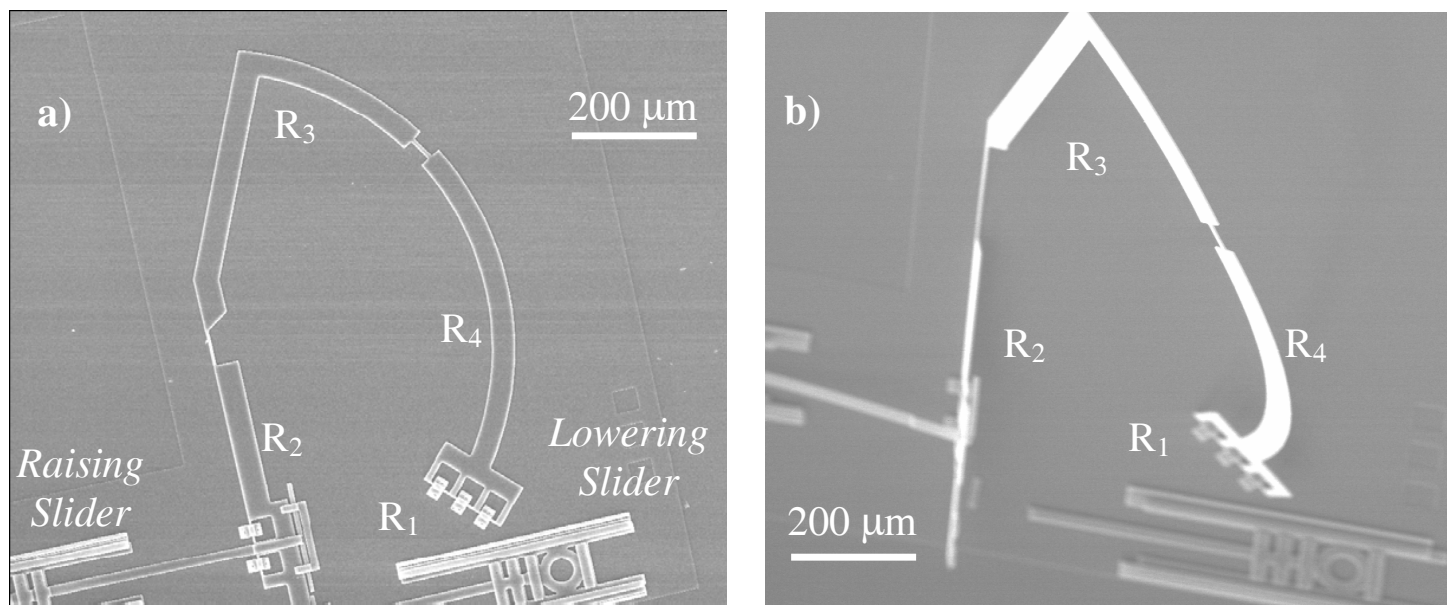

Figure 1. SEM micrograph of the Bistable Spherical Compliant Micromechanism (BSCM) in its stable positions: a) first stable position (as fabricated); b) second stable position (actuated).

Figure 1a is an SEM image of the MEMS device in its fabricated position. The Bistable Spherical Compliant Micromechanism (BSCM) has three basic components: two sliders and a compliant spherical four-bar mechanism with links $R_{1}, R_{2}, R_{3}$, and $R_{4} . R_{1}$ is the ground link, $R_{2}$ the input link, $R_{3}$ the coupler link and $R_{4}$ the follower link. Links $R_{2}$ and $R_{4}$ are joined to the substrate by a staple hinge [12] that allows $180^{\circ}$ rotation. Link $R_{3}$ is connected to $R_{2}$ and $R_{4}$ by compliant joints as shown in Figure 1a. The axes of rotation of the four joints intersect at a single point. The sliders act as mechanical actuators and are all connected to the input link $R_{2}$ by staple compliant hinges. The mechanism in its as-fabricated position is shown in Figure 1a, which is its first stable equilibrium position. By moving the Raising Slider to the left, link $R_{2}$ will rotate and links $R_{3}$ and $R_{4}$ will move out-of-plane. In order to bring the mechanism back to its original position, the Lowering Slider would be moved to the right. Figure $1 \mathrm{~b}$ shows the device in its second bistable position as actuated by the mechanical micromanipulator needle probe. The device was switched by the needle probe between the two bistable positions prior to scratch testing in the nanoindenter in order to avoid possible slider stiction. The lowering slider broke during the device actuation, and is no longer connected to the link $\mathrm{R}_{2}$.

In order to acquire the nonlinear mechanical response of the MEMS device, it was actuated by the Hysitron Triboindenter operating in the scratch mode. The Berkovich diamond tip was used in attempt to actuate the mechanism by scratching and moving the Raising Slider. The MEMS device was subject to two consequent displacement and normal force-controlled scratch tests. The normal force and lateral displacement profiles for the first test are shown in Figure 1. 


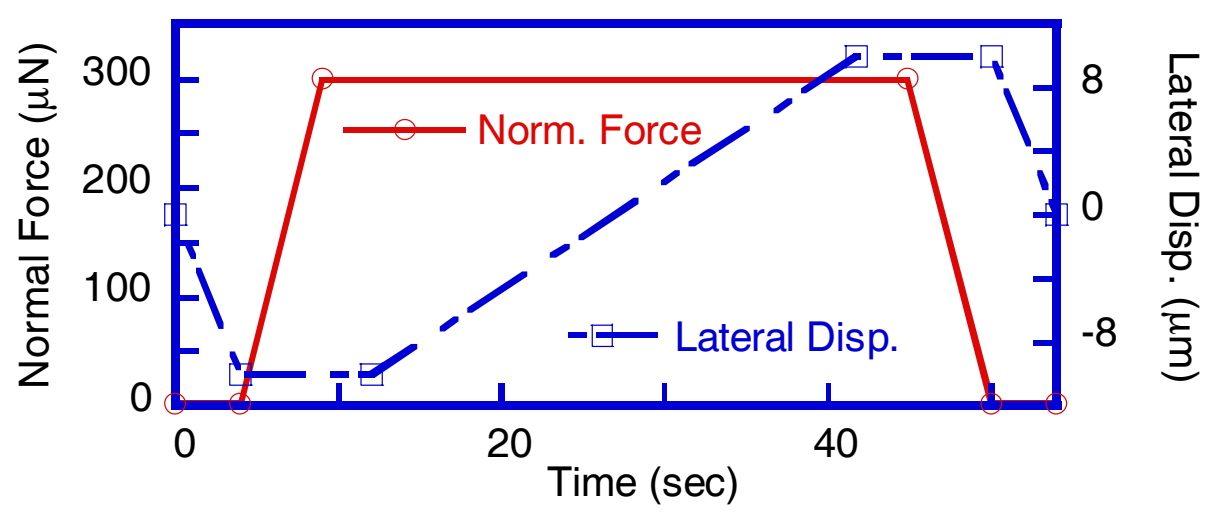

Figure 2. Normal force and lateral displacement profiles for the first scratch test.

In the first test, a maximum normal force of $300 \mu \mathrm{N}$ and a lateral displacement of 20 $\mu \mathrm{m}$ were applied to the Raising Slider (Figures 2 and 3), and in the second test the Raising Slider was subject to a normal force of $1000 \mu \mathrm{N}$ and a lateral displacement of $15 \mu \mathrm{m}$ (Figure 4).

\section{RESULTS}

The two test results are plotted in Figures 3 and 4. The plots in Figure 3 and Figure 4 have similar trends, even though they represent data taken from two different consequent tests performed on the same MEMS device. Figure 3a shows the lateral force and displacement of the indenter tip moving through the hole in the Raising Slider, and Figure $3 \mathrm{~b}$ shows the normal force and displacement.
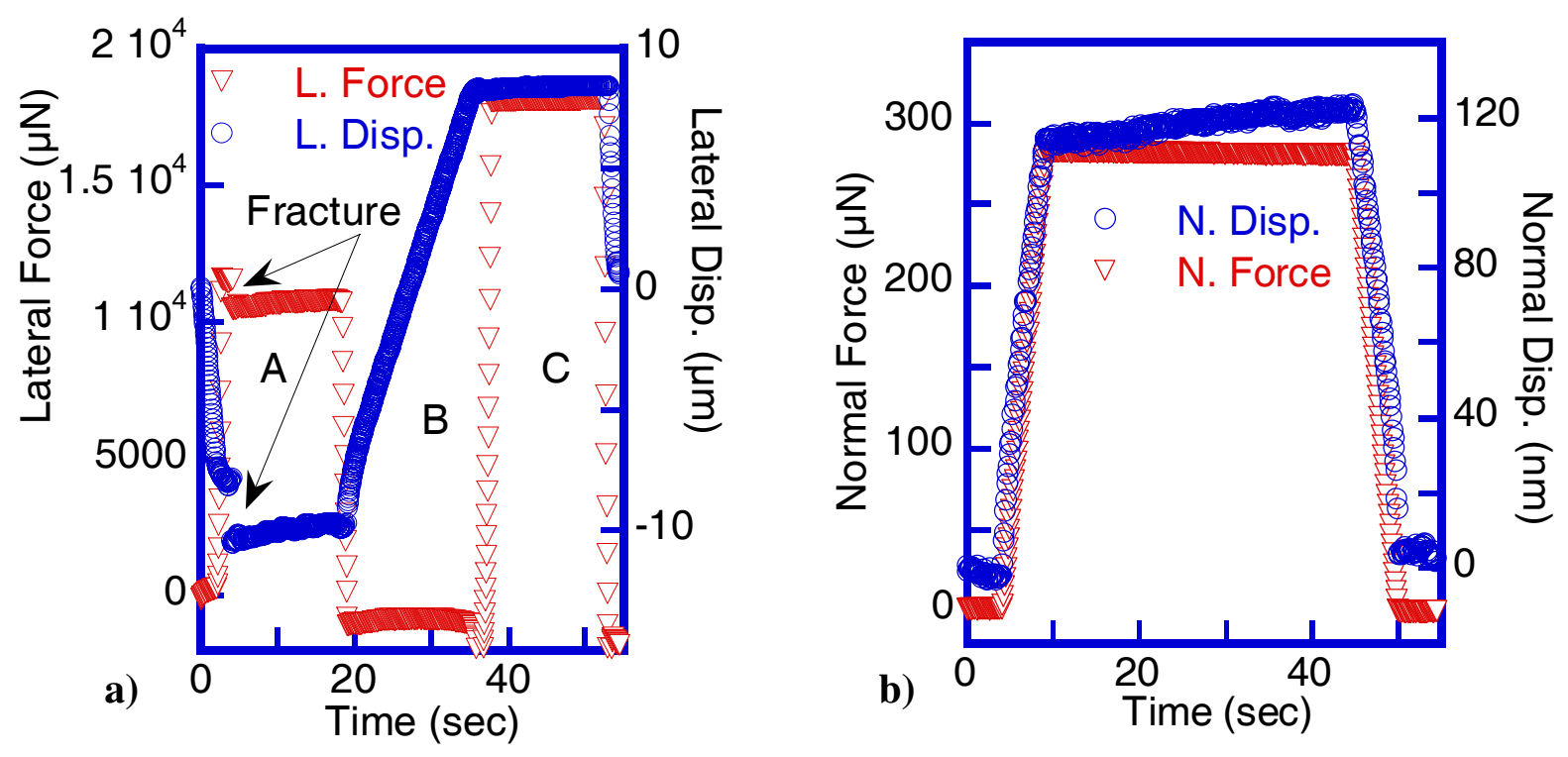

Figure 3. Test 1: a) Lateral force and displacement; b) normal force and displacement. 

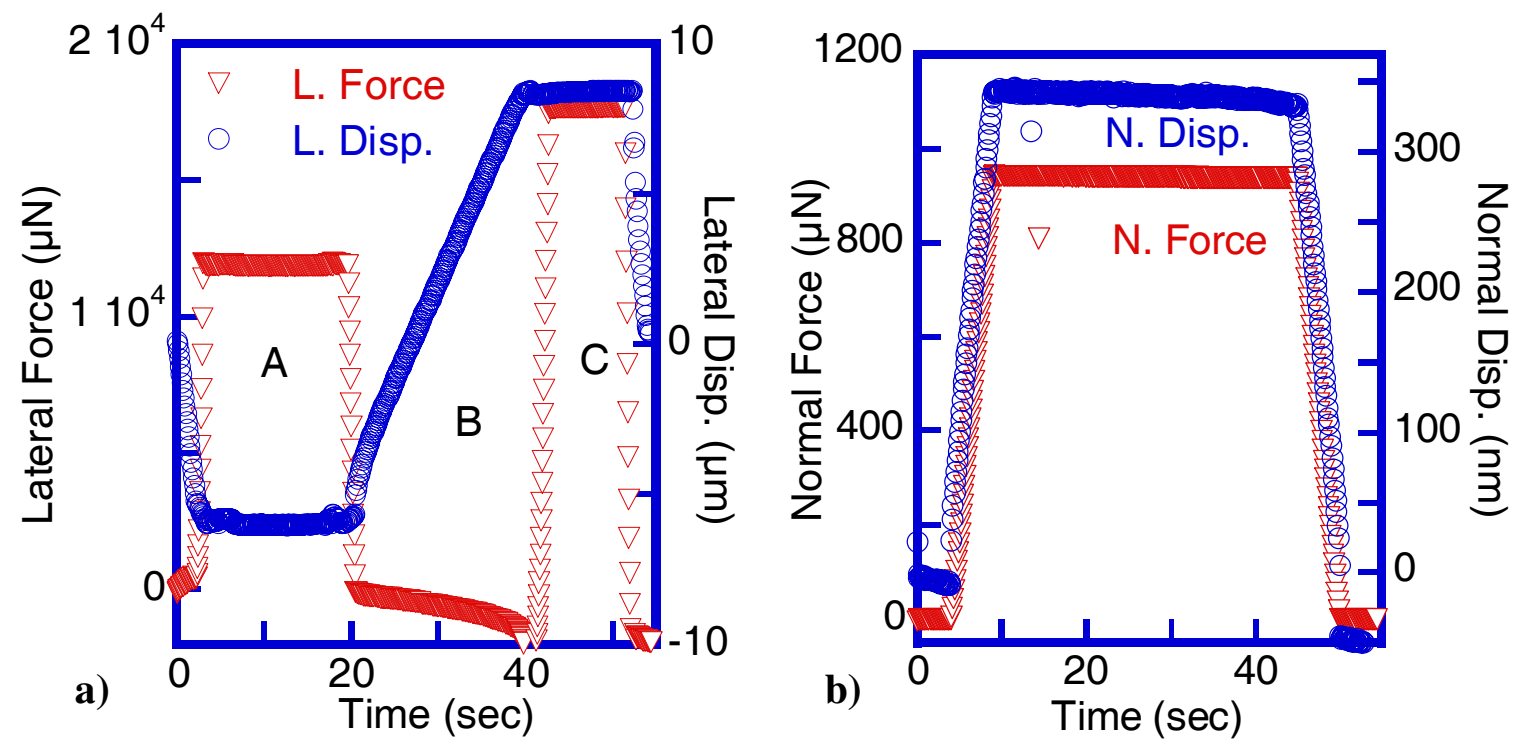

Figure 4. Test 2: a) Lateral force and displacement; b) normal force and displacement.

The plots obtained are significantly different form the expected cubic trend. The mechanical response of the MEMS mechanism could not be obtained. The lateral loads exerted on the Rising Slider by the indenter tip were not sufficient to cause the slider to move. On the other hand, the three-sided Berkovich tip edge was sharp enough that the force on the slider concentrated at a point caused its fracture due to high localized stresses. The slider fracture led to the discontinuity in displacement that occurred in part $A$ of Figures 3. Here, the fracture point is clearly identified by the lateral force and displacement discontinuity. The slider fractured during the portion of the scratch test in which the normal load started increasing (at $4 \mathrm{sec}$ ).

Figure 5 shows the lateral load-displacement measured by the indenter before and after the fracture in Test 1 . The initial $7.3 \mu \mathrm{m}$ of lateral displacement occurs with minimal lateral load and represents the motion of the indenter prior to its contact with the slider. A small stiffness of $34 \mathrm{~N} / \mathrm{m}$ is calculated from the pre-contact slope of the load-displacement curve. The stiffness increased to $26.5 \mathrm{kN} / \mathrm{m}$ when the indenter contacted the left side of the slider ring interior under the small normal pre-load of $2 \mu \mathrm{N}$. This stiffness is significantly higher than the stiffness of the transmission rod connecting the slider to the rest of the BSCM. The stiffness of the connecting rod is simply $k=E A / L$, which yields $10 \mathrm{kN} / \mathrm{m}$ for $E=$ $169 \mathrm{GPa}$, and $A=w t$, where $w=12 \mu \mathrm{m}, t=1.5 \mu \mathrm{m}$, and $L=275 \mu \mathrm{m}$. This implies that the resistance to the slider's motion did not come primarily from the BSCM, but from the forces between the slider and substrate and between the slider and the rails. The device underwent $0.5 \mu \mathrm{m}$ of lateral translation after the indenter tip contacted the slider and then when the normal force was increased, the slider fractured as evidenced by a rapid lateral displacement of about $2 \mu \mathrm{m}$ and a slight decrease in the lateral force resisting the motion of the indenter. The indenter then withdrew laterally from the slider, which gave an unloading stiffness of $51.6 \mathrm{kN} / \mathrm{m}$ over the $0.25 \mu \mathrm{m}$ lateral displacement. After this the indenter tip moved to the right inside the slider ring under high normal load. Due to the pyramidal tip geometry high normal applied force caused a lateral force component, which is represented in the negative lateral force in Figure 5a. Upon complete unloading the MEMS device exerts a lateral force of $2 \mathrm{mN}$ on the tip, which implies that the tip is in mechanical contact with the device. An optical micrograph of the non-damaged slider is shown in Figure $5 b$ for comparison with the one fractured during the test in Figure 5c. Closer slider inspection reveals chipped regions on 
the slider ring interior. The white triangle drawn in the center of the slider ring suggests the shape and the orientation of the indenter tip.
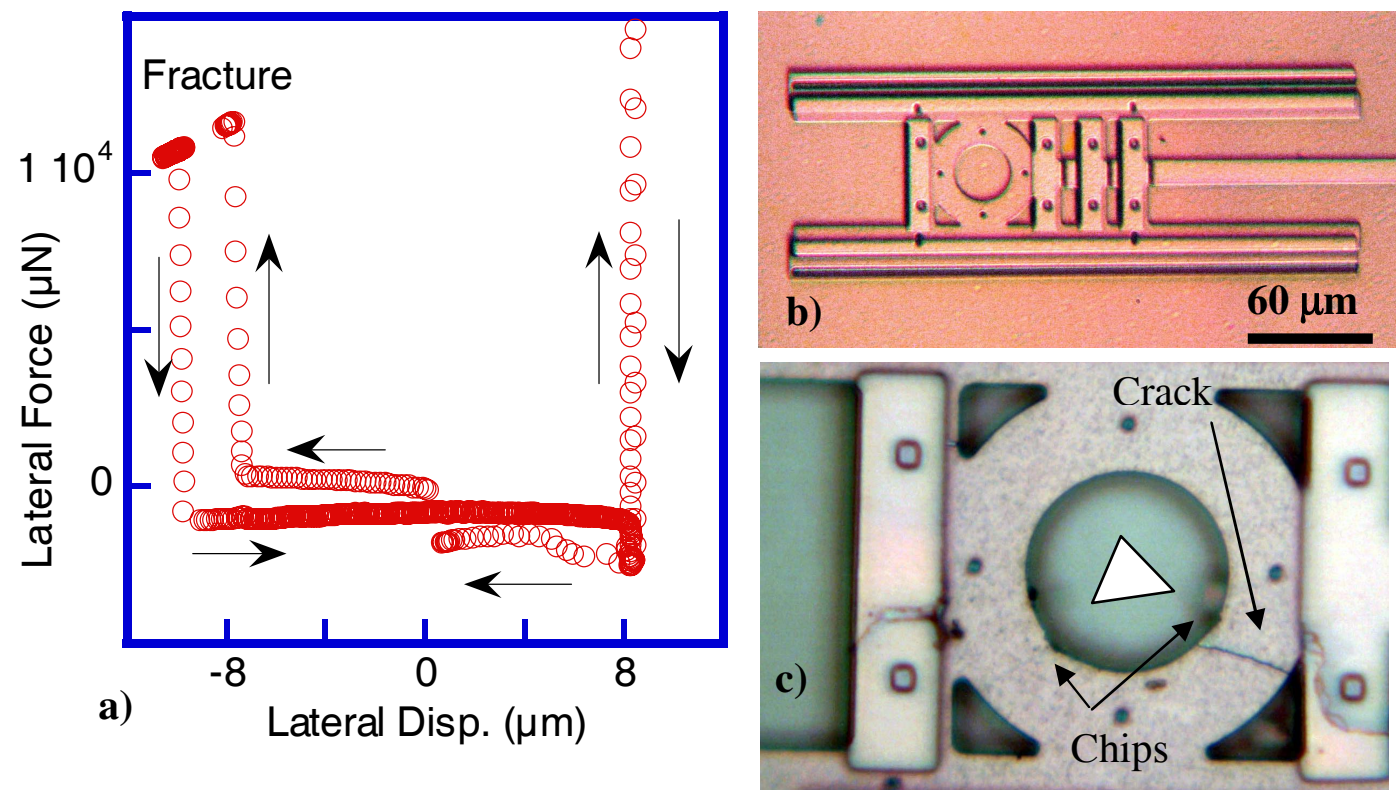

Figure 5. a) Lateral load-displacement profile showing slider fracture; b) nonactuated intact slider; c) Optical micrograph of the slider fracture. The white triangle represents the tip orientation. The inner slider ring diameter is $30 \mu \mathrm{m}$.

After leaving contact with the slider ring, the indenter tip moved across its interior and contacted the right side of the ring. On that side the stiffness measured by the indenter increased to $717 \mathrm{kN} / \mathrm{m}$, while the unloading stiffness was $992 \mathrm{kN} / \mathrm{m}$. The difference in stiffness of the slider ring opposite sides may be in part due to its geometry and in part due to the different orientation of the indenter tip when it came into contact with the other slider side.

Similar results where seen in the second test in which the normal force on the indenter tip was increased to a maximum of $1000 \mu \mathrm{N}$. A summary of the principal features of the load-displacement history seen in the tests is given in Table 1.

Table 1. Load-displacement history of the two consequent scratch tests.

\begin{tabular}{|l|l|l|}
\hline & Test 1 & Test 2 \\
\hline Max normal load & $300 \mu \mathrm{N}$ & $1000 \mu \mathrm{N}$ \\
\hline Left side loading stiffness & $26.5 \mathrm{kN} / \mathrm{m}$ & $24.6 \mathrm{kN} / \mathrm{m}$ \\
\hline Left side unloading stiffness & $51.6 \mathrm{kN} / \mathrm{m}$ & $56.6 \mathrm{kN} / \mathrm{m}$ \\
\hline Right side loading stiffness & $717 \mathrm{kN} / \mathrm{m}$ & $407 \mathrm{kN} / \mathrm{m}$ \\
\hline Right side unloading stiffness & $992 \mathrm{kN} / \mathrm{m}$ & $588 \mathrm{kN} / \mathrm{m}$ \\
\hline
\end{tabular}

The device loading stiffness is always less than its unloading stiffness, which implies that plastic deformation and/or fracture take place during loading, so the structure does not recover to its original shape. Thus, the structure reaches an unloaded condition with less displacement, which implies a higher stiffness.

The device was successfully switched between the two bistable positions with the needle probe, which has similar geometry to the conical indenter tip. In order to obtain an 
accurate representation of the mechanical response of the MEMS device, a conical indenter tip should be used, thus spreading the forces exerted on the slider and minimizing the risk of failure. In addition, the scratch displacement profile needs to be modified by removing the service segments $\mathrm{A}$ and $\mathrm{C}$ (designed for reducing drift while scratching a flat surface) to disable the tip reverse motion.

\section{CONCLUSIONS}

Force-displacement testing of a MEMS device was attempted using a nanoindenter. The localized stress caused by the Berkovich tip fractured the slider. The force required to actuate the device resulted in the stress concentration due to point contact between the sharp indenter tip and the slider, producing stresses that exceeded the fracture strength of the slider material. Load and displacement histories were recorded from the two tests, which reveal that the device requires a lateral force greater than $18 \mathrm{mN}$ for its actuation and that the loaddisplacement relationship for the slider ring of the BSCM device is affected by the normal force magnitude and the indenter tip geometry and orientation.

\section{ACKNOWLEDGEMENTS}

Alex Volinsky would like to acknowledge the support from NSF under CMMI0631526 and CMMI-0600231 grants. Xiaolu Pang would like to acknowledge the support from the State Scholarship Fund of China (No. 20063037).

\section{REFERENCES}

1. K. J. Gabriel, "Microelectromechanical Systems (MEMS) Tutorial”, IEEE Test Conference (TC), 432-441 (1998).

2. M. Mehregany and M. Huff, "Microelectromechanical Systems", Proceedings of the IEEE Cornell Conference on Advanced Concepts in High Speed Semiconductor Devices and Circuits, 9-18 (1995).

3. C. Lusk, "Ortho-Planar Mechanisms for Microelctromechanical Systems", Ph.D. Dissertation, Brigham Young University, Provo, UT (2005).

4. A.H.F Lam, W.J. Li, Yunhui Liu; Ning Xi, 2002 IEEE/RSJ International Conference on Intelligent Robots and System, vol. 2, 1184 - 1189 (2002).

5. T. Fukushige, S. Hata, A. Shimokohbe, Journal of Microelectromechanical Systems 14(2) 243-253 (2005).

6. G.K Ananthasuresh, L.L Howell, Larry, Journal of Mechanical Design 127(4) 736-738 (2005).

7. B. Felton, "Better Robots Through Clean Living", Intec, May 2001.

8. R. Cragun and L. L. Howell, "A New Constrained Thermal Expansion Micro-Actuator", American Society of Mechanical Engineers, Dynamic Systems and Control Division (Publication) DSC, vol. 66, 365-371 (1998).

9. C. D. Lott, J. Harb, T. W. McClain, L.L. Howell, Technical Proceedings of the Fourth International Conference on Modeling and Simulation of Microsystems, MSM 2001, Hilton Head Island, South Carolina, 374-377 (2001).

10. L.L Howell, A. Midha A., ASME Journal of Mechanical Design 116(1), 280-290 (1994).

11. D. Koester, R. Mahadevan, B. Hardy, and K. Markus, MUMPs Design Handbook. Research Triangle Park, NC: Cronos Integrated Microsystems (2001).

12. K.S.F Pister, M.W. Judy, S.R. Burgett, R.S. Fearing, Sensors and Actuators A, 33 249256 (1992). 